\title{
Partição de Comprimidos Antineoplásicos Utilizados no Tratamento de Leucemias Agudas em Crianças e Adolescentes
}

doi: https://doi.org/10.32635/2176-9745.RBC.2020v66n2.764

\author{
Splitting of Antineoplastic Tablets Used in Acute Leukemias Treatment in Children and Adolescents \\ Partición de Comprimidos Antineoplásicos Empleados en el Tratamiento de Leucemias Agudas en Niños y Adolescentes
}

Luiza Tessmann'; Patrícia Medeiros-Souzaㄹ; José Carlos Martins Cordoba ${ }^{3}$; Noemia Urruth Leão Tavares; Victória Macedo Abílio5; Dafny Oliveira de Matos ${ }^{6}$; Isis Maria Quezado Soares Magalhães ${ }^{7}$

Resumo

Introduçáo: A manipulação de antineoplásicos para ajuste de dose, como partição de comprimidos, é comum no tratamento de leucemias agudas de crianças e adolescentes. Objetivo: Identificar a frequência e descrever a prática da partição domiciliar de comprimidos antineoplásicos utilizados no tratamento oral de crianças e adolescentes com leucemias agudas na fase de manutenção. Método: Trata-se de um estudo transversal descritivo, realizado em um hospital pertencente à rede de saúde pública do Distrito Federal com assistência especializada em pediatria. Foram incluídos no estudo crianças e adolescentes entre 1 e 18 anos, diagnosticados com leucemias agudas e em fase de manutenção do tratamento no período de estudo. Foi aplicado um questionário semiestruturado ao responsável principal pela administraçáo dos medicamentos quimioterápicos via oral, podendo ser o cuidador ou a própria criança/adolescente. Foram coletadas variáveis sociodemográficas dos pacientes e cuidadores e variáveis sobre a prática de partição de medicamentos antineoplásicos no domicílio. Resultados: Todos os 48 entrevistados no período do estudo relataram ter partido comprimidos antineoplásicos ao longo do tratamento de leucemias agudas, sendo estes mercaptopurina $(n=45[93,75 \%])$ e tioguanina $(n=3$ [6,25\%]). Conclusáo: A partição de comprimidos antineoplásicos foi uma prática unânime em virtude da necessidade referida de ajuste de dose individual para o tratamento de leucemias agudas de crianças e adolescentes, considerando a indisponibilidade de formulaçóes adequadas. Os resultados reforçam a necessidade de a partiçáo ser uniformizada e realizada de maneira a minimizar os riscos e a garantir a segurança para as crianças e adolescentes e seus cuidadores.

Palavras-chave: Antineoplásicos/administração \& dosagem; Comprimidos/administração \& dosagem; Leucemia; Criança; Adolescente.

\begin{abstract}
Introduction: Antineoplastic drug manipulation for dose adjustment, such as tablet splitting, is standard in acute leukemia treatment for children and adolescents. Objective: To identify the frequency and describe the practice of household splitting of antineoplastic tablet for oral treatment of children and adolescents with acute leukemias in the maintenance phase. Method: Crosssectional descriptive study performed in a public health system hospital from Distrito Federal (Brazil) with specialized pediatric assistance. Children and teenagers between 1 and 18 years old, diagnosed with acute leukemia and in treatment maintenance phase during the study period were included. A semi-structured questionnaire was applied to the main responsible for administering oral chemotherapy drugs, which could be the caregiver or the child/adolescent themselves. Sociodemographic variables of patients and caregivers and variables on the practice of splitting antineoplastic drugs at home were collected. Results: All 48 interviewees in the study period reported having split antineoplastic tablets during the treatment for acute leukemias, such as mercaptopurine $(\mathrm{n}=45[93.75 \%])$ and thioguanine ( $\mathrm{n}$ $=3[6.25 \%])$. Conclusion: The splitting of antineoplastic tablets was a unanimous practice due to the reported need to adjust the individual dose for acute leukemia treatment in children and adolescents, considering the unavailability of adequate formulations. The results reinforce the need for splitting to be standardized and performed in a way that minimizes risks and ensures safety for patients and their caregivers.
\end{abstract}

Key words: Antineoplastic Agents/administration \& dosage; Tablets/ administration \& dosage; Leukemia; Child; Adolescent.
Resumen

Introducción: La manipulación de fármacos antineoplásicos para el ajuste de dosis, como las particiones de comprimidos, es frecuente en el tratamiento de las leucemias agudas en nińos y jóvenes. Objetivo: Identificar la frecuencia y describir la práctica de la división domiciliaria de medicamentos antineoplásicos utilizados en el tratamiento oral de niños y adolescentes con leucemias agudas en la fase de mantenimiento. Método: Se trata de un estudio transversal descriptivo realizado en un hospital de la red de salud pública del Distrito Federal (Brasil) con asistencia especializada en pediatría. El estudio incluyó a nińos y jóvenes de entre 1 y 18 años de edad diagnosticados con leucemia aguda y en fase de mantenimiento del tratamiento en el período del estudio. Se aplicó un cuestionario semiestructurado a la persona principal responsable de la administración de fármacos quimioterapéuticos por vía oral, que puede ser el cuidador o el propio niño/joven. Fueron colectadas variables sociodemográficas de los pacientes y cuidadores y variables sobre la práctica de la división de los medicamentos antineoplásicos en domicílios. Resultados: Los 48 entrevistados en el período de estudio informaron haber roto las pastillas antineoplásicas durante el tratamiento de la leucemia aguda, siendo éstas mercaptopurina $(n=45[93,75 \%])$ y tioguanina $(n=3[6,25 \%])$. Conclusión: La partición de comprimidos antineoplásicos fue una práctica unánime debido a la necesidad mencionada de ajustar la dosis individual para el tratamiento de las leucemias agudas de niños y adolescentes, considerando la falta de formulaciones apropiadas. Los resultados refuerzan la necesidad de estandarizar y realizar la partición para minimizar los riesgos y garantizar la seguridad de los niños, jóvenes y sus cuidadores.

Palabras clave: Antineoplásicos/administración \& dosificación; Comprimidos/ administración \& dosificación; Leucemia; Niño; Adolescente.

\footnotetext{
1 Universidade de Brasília (UnB). Faculdade de Ciências da Saúde (FS). Departamento de Farmácia. Brasília (DF), Brasil. Orcid iD: https://orcid.org/0000-0002-7157-164X ${ }^{2}$ UnB/FS/Departamento de Farmácia. Brasília (DF), Brasil. Orcid iD: https://orcid.org/0000-0003-4022-9187

${ }^{3}$ Secretaria de Estado de Saúde do Distrito Federal (SES/DF). Hospital da Criança de Brasília José Alencar. Brasília (DF), Brasil. Orcid iD: https://orcid.org/0000-0002-7573-1647

${ }^{4}$ UnB/FS/Departamentos de Farmácia e de Saúde Coletiva. Brasília (DF), Brasil. Orcid iD: https://orcid.org/0000-0001-6180-7527

${ }^{5}$ UnB/FS/Departamento de Farmácia. Brasília (DF), Brasil. Orcid iD: https://orcid.org/0000-0002-9986-6503

${ }^{6}$ UnB/FS/Departamento de Saúde Coletiva. Brasília (DF), Brasil. Orcid iD: https://orcid.org/0000-0003-3327-4262

${ }^{7}$ SES/DF. Núcleo de Oncologia e Hematologia Pediátrica no Hospital da Criança de Brasília. Hospital da Criança de Brasília José Alencar. Brasília (DF), Brasil. Orcid iD: https://orcid.org/0000-0003-2447-3753
}

Endereço para correspondência: Patrícia Medeiros-Souza. SQN 205, Bloco C, apartamento 403. Brasília (DF), Brasil. CEP 70843-030. E-mail: pmedeirossouza@uol.com.br 


\section{INTRODUÇÃO}

O câncer já representa a primeira causa de morte ( $8 \%$ do total) por doença no Brasil entre crianças e adolescentes de 1 a 19 anos $^{1}$. Os tumores mais frequentes entre as neoplasias pediátricas são as leucemias ${ }^{1}$. A leucemia é uma doença maligna de células hematopoiéticas e sua principal característica é o acúmulo de células doentes na medula óssea, que substituem as células sanguíneas normais². Existem mais de 12 tipos de leucemia, incluindo tipos primários agudos ${ }^{2}$.

O tratamento das leucemias agudas é divido em etapas e constitui-se de três fases principais: indução da remissão, consolidação e manutenção ${ }^{2}$. A última, fase de manutenção, caracteriza-se por tratamento mais brando e contínuo por vários meses ${ }^{2}$, com prescriçóes de medicamentos para uso via oral e domiciliar, sob responsabilidade da família.

A terapia de manutenção é tão importante quanto as fases iniciais de tratamento ${ }^{3}$ para que se obtenha cura e não haja recidiva ${ }^{2}$. $\mathrm{O}$ uso correto de quimioterápicos e o melhor cuidado de suporte contribuíram para melhorar o tratamento e aumentaram as taxas de sobrevida ao longo dos anos ${ }^{4,5}$. O ajuste de dose individual adequado é importante para suprimir o ressurgimento do clone leucêmico e para diminuir a toxicidade por leucopenia ${ }^{3}$, levando a um melhor resultado do tratamento e à cura ${ }^{4}$.

A manipulaçáo de medicamentos na pediatria para ajuste de dose individual, incluindo a partição de comprimidos, é algo comum e comprimidos divididos são frequentemente usados na prática clínica para atingir a dose prescrita ${ }^{6-8}$. A falta de disponibilidade de variadas formas farmacêuticas de quimioterápicos, utilizados para o tratamento de leucemias agudas de crianças e adolescentes, faz com que esses medicamentos sejam manipulados para atender às demandas pediátricas ${ }^{9,10}$.

Isso pode ser problemático, considerando que grande parte dos antineoplásicos são citotóxicos e agentes perigosos, podendo causar danos às pessoas que estão envolvidas na preparação, na administração de medicamentos e no cuidado de quem está em quimioterapia ${ }^{11-14}$, já que a partição de comprimidos em um ambiente não controlado as expóe à poeira citotóxica ${ }^{10}$. Além disso, a manipulação também é preocupante por conta de questôes que incluem estabilidade, biodisponibilidade e precisão da dosagem do medicamento, podendo ser administradas doses tóxicas ou subterapêuticas para a criança/adolescente $e^{6,8}$. Isso se torna mais relevante levando em consideraçáo que os quimioterápicos têm um índice terapêutico estreito, então a administração correta é crucial para que se tenha eficácia e segurança ${ }^{15}$.
Há uma lacuna de conhecimento em relação à partição domiciliar dos comprimidos antineoplásicos e à forma como é realizada. Além disso, dados a respeito da contaminação e exposição a essas drogas perigosas em ambientes fora de hospitais também são $\operatorname{raros}^{14}$. Isso se torna relevante, pois engloba fatores que precisam ser melhor estudados, desde a segurança do paciente e do cuidador/familiar, além da eficácia do tratamento e dos possíveis eventos adversos tardios provenientes do processo de partição domiciliar.

Dessa forma, o objetivo do presente estudo foi identificar a frequência de partiçáo domiciliar dos comprimidos antineoplásicos utilizados no tratamento oral de crianças e adolescentes com leucemias agudas em fase de manutenção atendidos em um hospital pediátrico e descrever a prática de partição, a forma como é realizada, para compreender melhor o processo, tendo em vista os riscos que esses medicamentos podem causar para quem os utiliza e quem os manipula.

\section{MÉTODO}

Trata-se de um estudo transversal descritivo, realizado no período de janeiro a março de 2019, em um hospital público de referência em pediatria localizado em Brasília, DF.

O fluxograma (Figura 1) apresenta a seleção dos participantes do estudo, que se deu a partir da lista dos pacientes onco-hematológicos atendidos no local e de consulta em prontuário eletrônico. Das 82 crianças e adolescentes diagnosticados com leucemias agudas de novo ou recaída, que estavam em tratamento no período de estudo, foram incluídos crianças e adolescentes com idade entre 1 a 18 anos, que atingiram a fase de manutenção da quimioterapia com prescrição de uso contínuo de medicamentos quimioterápicos orais, sob regime domiciliar e que estavam acompanhados pelo

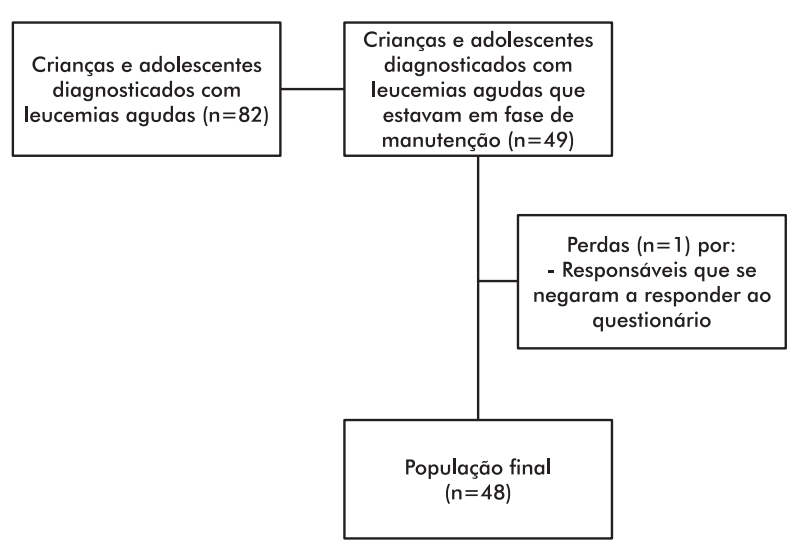

Figura 1. Fluxograma da seleção dos participantes do estudo 
responsável principal dos cuidados com a criança ou adolescente no período do estudo $(n=49)$.

A coleta de dados foi realizada por meio da aplicação de um questionário semiestruturado, na forma de entrevista, sobre a prática de partição dos antineoplásicos, ao responsável principal pela administração dos medicamentos, ao cuidador ou à própria criança/ adolescente, em local reservado de espera da consulta no ambulatório do hospital.

Foram levantadas variáveis sociodemográficas de todas as crianças/adolescentes e de seus respectivos cuidadores. As variáveis sociodemográficas das crianças/adolescentes incluíram sexo, idade, cor da pele e acesso a planos de saúde. Já as dos cuidadores incluíram sexo, idade, escolaridade, estado civil, cor da pele, renda familiar e grau de parentesco com o paciente. De forma complementar, foram levantadas informaçóes a partir dos prontuários eletrônicos, como histórico da condição patológica e medicamentos em uso dos pacientes.

Em relaçáo à prática da partiçáo domiciliar de antineoplásicos orais, foram coletadas, por meio do questionário, as seguintes variáveis: se os entrevistados partiam ou já tiveram que partir ao longo do tratamento e as condiçóes desse processo de partição. Sendo assim, foram investigados qual o instrumento utilizado, qual o local da casa em que se partia, como era feita a armazenagem das outras partes do comprimido, alteraçôes dos comprimidos partidos relatadas pelos entrevistados, higienização do manipulador e do instrumento utilizado e sintomas percebidos pelo responsável pela partição após a manipulação do antineoplásico. Considerando a prática atual de partição, analisou-se em quantas partes eram partidos os comprimidos (metades, terços ou quartos), levando em conta a capacidade de recordação dos entrevistados e a importância de demonstrar a partição domiciliar naquele momento.

A partir dos dados coletados, foi construído um banco de dados por meio do programa Epi Info versáo 7.0 e realizada a análise estatística. $\mathrm{Na}$ análise descritiva, os dados foram expressos como frequência absoluta ou relativa e medidas de tendência central e de variabilidade.

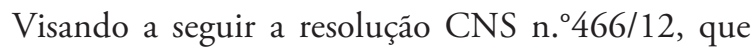
dispóe sobre as Diretrizes e Normas Regulamentadoras de Pesquisas Envolvendo Seres Humanos, a pesquisa foi submetida à apreciação e aprovada pelo Comitê de Ética em Pesquisa com Seres Humanos da Faculdade de Ciências da Saúde da Universidade de Brasília (UnB), de acordo com o número CAAE: 87652818.5.0000.0030 e o parecer de aprovação número 2.808.180, em 11 de agosto de 2018, e pelo Comitê de Ética em Pesquisa da Fundaçáo de Ensino e Pesquisa em Ciências da Saúde (Fepecs), com o número CAAE: 87652818.5.3001.5553 e parecer de aprovação número 2.979.001, em 24 de outubro de 2018. As entrevistas foram realizadas após a assinatura do Termo de Consentimento Livre e Esclarecido (TCLE) pelos cuidadores responsáveis e do Termo de Assentimento Livre e Esclarecido (TALE) pelas crianças, a partir de cinco anos de idade, e adolescentes.

\section{RESULTADOS}

No total, foram entrevistados 48 responsáveis pela administração dos medicamentos antineoplásicos, 45 [93,75\%]) eram os cuidadores das crianças e adolescentes, sendo que, em 40 casos $(85,42 \%)$, a máe era a principal responsável pelo tratamento e, em três casos $(6,25 \%)$, quem respondeu ao questionário sobre a partição foi o próprio adolescente. Em relação às características sociodemográficas dos cuidadores $(\mathrm{n}=48)$ conforme a Tabela 1, observa-se que a média etária foi de 33,56 anos $(\mathrm{DP}=9,66)$, sendo que a maioria era do sexo feminino $(\mathrm{n}=44[91,67 \%])$ e de pele parda $(\mathrm{n}=27[56,25 \%])$. O maior percentual de cuidadores era casado $(\mathrm{n}=17$ [35,42\%]), tinha ensino médio completo ( $\mathrm{n}=18$ [37,5\%]) e renda média familiar variando entre um e dois salários mínimos (n=29 [60,43\%]).

Quanto aos dados alusivos a crianças/adolescentes $(\mathrm{n}=48)$, constatou-se predominância de pele parda $(\mathrm{n}=20$ $[41,67 \%])$ e do sexo masculino $(\mathrm{n}=32[66,67 \%])$, com média etária de 6,71 anos $(\mathrm{DP}=3,81)$. Observou-se hegemonia da leucemia linfoide aguda $(\mathrm{n}=47$ [97,92\%]) sobre a leucemia mieloide aguda $(\mathrm{n}=1[2,08 \%])$. Outro dado relevante é que a maior parte das crianças e adolescentes náo possuía vínculos a planos de saúde $(\mathrm{n}=42[87,50 \%])$.

A partir dos dados levantados, observou-se no estudo que os antineoplásicos via oral de uso contínuo utilizados pelas crianças e adolescentes na fase de manutenção de leucemias agudas foram metotrexato $(n=41[85,42 \%])$, desatinibe $(n=1[2,08 \%])$, mercaptopurina $(n=45$ $[93,75])$ e tioguanina $(n=3[6,25 \%])$. Os resultados demonstraram que os 48 entrevistados relataram já ter partido comprimidos antineoplásicos ao longo do tratamento, sendo estes mercaptopurina $50 \mathrm{mg}$ e tioguanina $40 \mathrm{mg}$. A partição de mercaptopurina havia sido realizada por 45 entrevistados $(93,75 \%)$, enquanto a de tioguanina por três $(6,25 \%)$, no momento das entrevistas ou previamente. Dos 48 entrevistados, ressalta-se que $33(68,75 \%)$ estavam realizando partição domiciliar no momento da entrevista para ajuste de dose individual (Tabela 2).

Um aspecto avaliado foi em quantas partes os comprimidos foram partidos para atingir a dose necessária. Para isso, a análise considerou a partição domiciliar 
Tabela 1. Dados sociodemográficos dos cuidadores das crianças e adolescentes em tratamento de leucemias agudas

\begin{tabular}{|c|c|c|}
\hline Dados demográficos & Cuidadores (n) & Frequência (\%) \\
\hline \multicolumn{3}{|l|}{ Sexo } \\
\hline Feminino & 44 & 91,67 \\
\hline Masculino & 4 & 8,33 \\
\hline \multicolumn{3}{|l|}{ Idade (anos) } \\
\hline De 18 até 27 & 16 & 33,33 \\
\hline De 28 até 37 & 16 & 33,33 \\
\hline De 38 até 47 & 11 & 22,92 \\
\hline De 48 até 57 & 5 & 10,42 \\
\hline \multicolumn{3}{|l|}{ Cor da Pele } \\
\hline Branca & 7 & 14,58 \\
\hline Amarela & 8 & 16,67 \\
\hline Preta & 5 & 10,42 \\
\hline Indígena & 1 & 2,08 \\
\hline Parda & 27 & 56,25 \\
\hline \multicolumn{3}{|l|}{ Estado civil } \\
\hline Solteiro (a) & 11 & 22,92 \\
\hline Casado (a) & 17 & 35,42 \\
\hline União estável & 13 & 27,08 \\
\hline Divorciado (a) & 7 & 14,58 \\
\hline \multicolumn{3}{|l|}{ Escolaridade } \\
\hline Não sabe ler ou escrever & 1 & 2,08 \\
\hline Ensino fundamental completo & 3 & 6,25 \\
\hline Ensino fundamental incompleto & 12 & 25,00 \\
\hline Ensino médio completo & 18 & 37,50 \\
\hline Ensino médio incompleto & 8 & 16,66 \\
\hline Ensino superior completo & 2 & 4,17 \\
\hline Ensino superior incompleto & 2 & 4,17 \\
\hline Pós-graduação completa & 2 & 4,17 \\
\hline \multicolumn{3}{|l|}{ Renda familiar } \\
\hline Até 1 salário & 6 & 12,50 \\
\hline De 1 até menos de 2 salários ( $R \$ 788,00$ a $R \$ 1.575 .99$ ) & 29 & 60,43 \\
\hline De 2 até menos de 4 salários ( $R \$ 1.576 .00$ a $R \$ 3.151 .99$ ) & 7 & 14,58 \\
\hline De 4 até menos de 6 salários ( $R \$ 3.152 .00$ a $R \$ 4.727 .99$ ) & 4 & 8,33 \\
\hline De 6 até menos de 10 salários ( $R \$ 4.728 .00$ a $R \$ 7.879 .99$ ) & 1 & 2,08 \\
\hline 10 salários ou acima ( $\geq R \$ 7.880,00)$ & 1 & 2,08 \\
\hline
\end{tabular}

no momento das entrevistas por conta de capacidade de recordação dos entrevistados e pela importância de representar a partição naquele momento. Os antineoplásicos foram partidos em metades, quartos ou das duas formas, dependendo da prescrição para ajuste de dose conforme o dia. Esses dados estáo detalhados na Tabela 2.
A análise das condiçôes do processo de partição de comprimidos antineoplásicos levou em consideração também a partiçấo domiciliar realizada previamente ao momento das entrevistas, visto que todos relataram já ter partido ao longo do tratamento das leucemias agudas. Conforme a Tabela 3, o principal instrumento utilizado 
para a partição foi o partidor de comprimidos $(n=23$ $[47,92 \%])$, a cozinha foi o principal local de partição $(n=37[77,09 \%])$. Os 48 entrevistados armazenavam as partes para uso posterior, sendo que a maioria $(n=38$ [79,17\%]) relatou armazenar na própria embalagem, contendo os comprimidos inteiros e partidos.

Tabela 2. Partição dos comprimidos de mercaptopurina e tioguanina utilizados por crianças e adolescentes em tratamento com leucemias agudas, no momento das entrevistas

\begin{tabular}{|c|c|c|}
\hline $\begin{array}{l}\text { Partição do } \\
\text { comprimido }\end{array}$ & $\begin{array}{l}\text { Entrevistados } \\
\text { (n) }\end{array}$ & $\begin{array}{c}\text { Frequência } \\
\text { (\%) }\end{array}$ \\
\hline \multicolumn{3}{|l|}{ Mercaptopurina } \\
\hline Sem partição & 15 & 31,25 \\
\hline Metades (1/2) & 14 & 29,17 \\
\hline Quartos (1/4) & 7 & 14,58 \\
\hline $\begin{array}{l}\text { Metades e } \\
\text { quartos }(1 / 2 \text { e } 1 / 4)\end{array}$ & 9 & 18,75 \\
\hline \multicolumn{3}{|l|}{ Tioguanina } \\
\hline Sem partição & 0 & 0,0 \\
\hline Metades (1/2) & 0 & 0,0 \\
\hline Quartos (1/4) & 2 & 4,17 \\
\hline $\begin{array}{l}\text { Metades e } \\
\text { quartos }(1 / 2 \text { e } 1 / 4)\end{array}$ & 1 & 2,08 \\
\hline
\end{tabular}

Outros fatores a respeito das condições de partição incluíram percepção de alteração nos comprimidos partidos e higiene. Em relação a alteraçôes, 16 entrevistados $(33,33 \%)$ relataram percepção de esfarelamento dos comprimidos após a partição. E, quanto à higiene, todos disseram lavar as mãos antes de partirem os comprimidos, porém, sete $(14,58 \%)$ declararam não as higienizar após a partição. E em relação à higiene dos instrumentos, 32 entrevistados $(66,67 \%)$ relataram limpá-los antes e depois de os utilizarem para partir os medicamentos e quatro $(8,33 \%)$ limpavam o instrumento somente antes da partição. Alguns entrevistados ( $\mathrm{n}=12$ [25\%]) declararam não realizar essa higiene nem antes e nem depois de cada partição. Houve ainda a preocupação em identificar se a pessoa responsável por partir sentiu algum sintoma ao realizar a divisão dos comprimidos, dois $(4,17 \%)$ entrevistados relataram coceira ao partirem os comprimidos de mercaptopurina, enquanto um entrevistado (2,08\%) apresentou dor de cabeça e náusea ao partir esse medicamento.

\section{DISCUSSÃO}

Este estudo sobre partição de antineoplásicos de via oral utilizados no tratamento de manutenção de leucemias agudas por crianças e adolescentes revelou que os 48 entrevistados já tiveram que realizar a partição em algum momento para ajuste de dose individual. O cálculo de

Tabela 3. Condições do processo de partição dos comprimidos antineoplásicos

\section{Processo de partição}

Entrevistados Frequência

(n)

(\%)

\section{Instrumento utilizado}

Partidor de comprimidos

Faca

Mão

Estilete

Local de realização da partição dos comprimidos

Cozinha

Quarto

Local indefinido

\section{Como são armazenados os comprimidos partidos}

Frasco contendo comprimidos partidos e inteiros do próprio antineoplásico

Frasco contendo só comprimidos partidos do próprio antineoplásico

Outros potes e porta-comprimidos 
doses dos antineoplásicos é inicialmente realizado por intermédio do valor superfície corporal $\left(\mathrm{m}^{2}\right)$, mas deve ser ajustado de acordo com a leucometria ${ }^{3,16,17}$ por possuírem índice terapêutico relativamente estreito e toxicidade potencialmente fatal, principalmente em forma de mielossupressão ${ }^{18,19}$, tornando a prescrição individualizada.

Os comprimidos antineoplásicos partidos foram mercaptopurina $50 \mathrm{mg}$ e tioguanina $40 \mathrm{mg}$, os quais são análogos dos nucleosídeos de purina utilizados no tratamento de leucemias agudas ${ }^{18-20}$. A prática de manipulação desses quimioterápicos para ajuste de dose individual se torna comum ${ }^{9}$ tendo em vista a falta de formulações adequadas disponíveis ${ }^{21,22}$. Órgãos regulatórios de alguns países já aprovaram a comercialização de uma suspensão oral de mercaptopurina ${ }^{10,17,23}$, porém ainda não está disponível amplamente, não sendo então uma realidade brasileira. A gravidade de leucemias agudas na infância e os problemas associados à dosagem correta de quimioterápicos exigem formulaçôes pediátricas orais adequadas ${ }^{10,24}$, porque, apesar de ser uma doença grave, apresenta alta taxa de cura e sobrevida se o tratamento for realizado corretamente $e^{4,5}$.

Os resultados demonstraram que, no momento das entrevistas, 33 (68,75\%) entrevistados estavam partindo esses comprimidos em domicílio para atingir as doses-alvo terapêuticas. Pode ser observado que, por vezes, foi necessário mais de uma partição para poder atingir quartos. $\mathrm{Na}$ literatura, se discute que quanto mais vezes se realizar a partição de um comprimido, como para atingir quartos, maior será a sua variabilidade de massa e perda de peso do comprimido, comprometendo a uniformidade ${ }^{7,8,25}$. Sendo assim, a maior probabilidade de partes com tamanhos irregulares leva à flutuação e à imprecisáo da dose $\mathrm{d}^{7,26}$. Nas prescrições em que é necessário partir de mais de uma forma (metades e quartos), pode ocorrer tanto uma diminuição de eficácia quanto aumento da toxicidade em razão da confusão das partes que devem ser administradas a cada dia, considerando que a partição de comprimidos pode gerar erros de medicação ${ }^{24}$.

A partição dos comprimidos de mercaptopurina e tioguanina é preocupante, levando em consideraçấo que são citotóxicos, considerados agentes perigosos, e que possuem margem de segurança relativamente estreita ${ }^{11,12,17,27,28}$. A contaminação pode ser por inalação, via oral e tópica ${ }^{11,12,17,28}$. Dessa forma, foram avaliadas as condiçóes do processo de partição desses medicamentos antineoplásicos.

Em relação à percepção de alterações nos comprimidos antineoplásicos partidos, os resultados demonstraram que 16 entrevistados $(33,33 \%)$ relataram esfarelamento dos comprimidos, o que é potencialmente prejudicial ao ambiente doméstico ${ }^{29}$. O esfarelamento interfere na variação de peso do comprimido que, segundo Teixeira et $a^{30} .^{30}$ é um dos índices mais importantes para definir a segurança de um processo de subdivisão porque é relacionado diretamente à dose quando a substância ativa está uniformemente distribuída na massa do comprimido $^{30}$. Neste estudo, é ainda mais relevante por serem quimioterápicos citotóxicos ${ }^{11,12,17,27,28}$, que podem ocasionar contaminação e risco à saúde para os responsáveis pela partição ${ }^{25}$, bem como para quem utiliza os medicamentos. De acordo com o estudo experimental de Breitkreutz et al. ${ }^{29}$ para comparar métodos utilizados para preparações extemporâneas de mercaptopurina para crianças, até $0,46 \%$ da massa total do comprimido de mercaptopurina é perdida e liberada a cada procedimento de divisão, em forma de poeira citotóxica.

Um fator importante que pode afetar no esfarelamento, quando se realiza a partição dos comprimidos, é o instrumento. Os resultados demonstraram que os principais instrumentos utilizados para partir os antineoplásicos foram: partidor de comprimidos, faca e máos. Os partidores de comprimidos são seguros e fáceis de usar ${ }^{31}$, porém é necessária destreza para posicionar o comprimido no local certo, evitando que fique com partes de tamanhos irregulares ${ }^{26}$. Além disso, o partidor, por apresentar uma lâmina afiada, necessita de maior atenção e cuidado ao ser manipulado ${ }^{25}$. Estudo experimental comparando a utilizaçáo de partidor de comprimidos e faca demonstrou que a variação de peso dos comprimidos partidos é menor entre os fragmentos obtidos por partidor, comparados àqueles obtidos com uma faca ${ }^{30}$. O estudo experimental de Habib et al. ${ }^{31}$ avaliou a precisão da divisão comparando a utilização de partidor de comprimidos versus das mãos para partir comprimidos de salbutamol $4 \mathrm{mg}$ e demonstrou que o uso de um partidor é mais preciso e superior à divisão manual, pois leva a uma menor variaçáo de peso e de teor do fármaco entre as partes. Partes de comprimidos divididos à máo podem se desviar em mais de $20 \%$ do seu peso ideal ${ }^{32}$, representando risco para a uniformidade do comprimido ${ }^{31}$. Sendo assim, na literatura se discute que, quando a partiçáo não puder ser evitada, a forma mais indicada para partir comprimidos é utilizando o partidor, por levar a uma menor variação e perda de peso do que os outros métodos de partiçáo $0^{33}$.

O local onde os antineoplásicos sáo partidos também foi um fator importante. Os resultados demonstraram que a maioria dos entrevistados partia na cozinha, o que é prejudicial, uma vez que os resíduos dos comprimidos partidos podem se misturar a alimentos e causar intoxicaçáo às pessoas que os ingerirem ${ }^{17,28}$. Então, em áreas onde os quimioterápicos são administrados e manuseados, deve-se evitar comer, beber e manter alimentos ${ }^{28}$.

Outro fator que deve ser considerado ao partir os comprimidos antineoplásicos é o armazenamento 
das partes náo utilizadas. Todos os entrevistados relataram armazenar as fraçóes para uso posterior. Retirar comprimidos da embalagem ao partir pode acelerar a degradação do princípio ativo, e, como consequência, levar ao recebimento de uma dose menor, além de potencializar efeitos adversos ${ }^{26}$. Pode ocorrer um aumento do risco de problemas de estabilidade e de imprecisão na dosagem quando muitos comprimidos partidos são armazenados dentro de uma embalagem ${ }^{25,32,34}$, por conta do atrito entre as fraçóes, o que ocasiona um maior grau de esfarelamento dos medicamentos ${ }^{34}$. A literatura relata que a maneira mais indicada para armazenar a fração que será utilizada posteriormente é guardá-la na própria embalagem, a fim de minimizar sua exposição ao ambiente ${ }^{34,35}$. Em relação aos porta-comprimidos e a outros potes, estes podem levar a uma exposição à umidade ambiental e à luz ${ }^{34,35}$, bem como ao armazenar no próprio partidor de comprimidos. $\mathrm{O}$ armazenamento em geral traz questóes preocupantes em relação ao tempo que os comprimidos divididos são expostos ao ar e à luz antes do uso ${ }^{26}$.

A higiene do manipulador dos antineoplásicos e do instrumento que se utilizava para partir também foi analisada, sendo importante ressaltar que, quando o contato dérmico com quimioterápicos não pode ser evitado, deve-se aderir a rigorosas medidas de higiene ${ }^{14}$. Os resultados demonstraram que todos os entrevistados higienizavam as mãos antes de partirem os comprimidos para garantir a segurança das crianças e adolescentes, mas alguns não as lavavam após a divisão. A higiene após o processo de partição também é importante, pois há a possibilidade de intoxicação decorrente do manuseio desses medicamentos tóxicos, já que podem entrar no corpo por meio de inalaçáo, absorção dérmica e contato pela boca ${ }^{11,12,17,28,34}$ e têm potencial para causar câncer, toxicidade e danos aos órgãos ${ }^{11,12,17,28}$, representando risco para quem os manipula. Mãos contaminadas podem transferir resíduos para a boca e outras superfícies $^{28}$. É necessário lavar as mãos após manusear os antineoplásicos, se possível até utilizar equipamentos de proteção individual, como luvas ${ }^{11-14,17,28}$, para evitar contato dérmico e contaminação por ingestáo e inalação. Medidas higiênicas e protetoras em geral devem ser incorporadas ao ambiente doméstico, para minimizar o risco a todos ${ }^{14}$.

Em relaçáo à higiene dos instrumentos utilizados na partição dos antineoplásicos, é importante ressaltar que parte dos entrevistados relatou não os higienizar nem antes e nem depois do processo de partiçáo e alguns os limpavam somente antes. A falta de limpeza pode levar à contaminaçáo cruzada causada por esfarelamento, se forem partidos diferentes medicamentos com o mesmo instrumento ${ }^{25,32}$.
Em relação aos sintomas, foram relatados coceira, dor de cabeça e náusea por alguns entrevistados. Nesse enfoque, a exposição a drogas perigosas pode resultar em efeitos adversos, agudos e crônicos como erupçóes cutâneas, resultados reprodutivos adversos e até câncer ${ }^{11,12,17,27,28,36}$.

Formulaçôes extemporâneas preparadas na pediatria podem ter vantagens sobre a partição de comprimidos ${ }^{7}$. Sendo assim, como possível estratégia para evitar a partição domiciliar dos antineoplásicos, poderia ocorrer o preparo extemporâneo de formulaçôes líquidas em suspensão de mercaptopurina e tioguanina ${ }^{17} \mathrm{em}$ farmácias de manipulação especializadas em quimioterapia. Medidas de proteção necessitam ser tomadas para a segurança dos profissionais, como manipulação em cabines de segurança biológica ${ }^{17,36}$; e estudos adicionais de estabilidade precisam ser realizados para garantir a eficácia do medicamento, pois esse tipo de formulação pode envolver problemas nesse quesito ${ }^{25,29}$. Porém, o ideal seriam formulaçôes líquidas dos antineoplásicos disponíveis comercialmente de maneira ampla.

Em relação às limitaçóes do estudo, cabe destacar o desenho transversal, que impede a definição de causalidade. A quantidade de crianças/adolescentes e cuidadores entrevistados também pode ser considerada um fator limitante, além de a pesquisa ter sido realizada em um único serviço de saúde. Por fim, a comparação foi feita com estudos experimentais, tendo em vista a falta de estudos clínicos que abordassem a partição domiciliar de antineoplásicos na pediatria.

\section{CONCLUSÃO}

A investigação da frequência de partição de comprimidos antineoplásicos mostrou que todos os entrevistados já a realizaram para ajuste de dose individual. Quando a partição não puder ser evitada, como no caso desses quimioterápicos em estudo, em razão da falta de formulaçóes adequadas, é importante uniformizar essa prática, diminuindo o risco para as crianças e adolescentes e para os cuidadores. Informaçôes sobre manuseio seguro de antineoplásicos devem ser incorporadas nos serviços de saúde. Além disso, ressalta-se a importância de mais estudos e investimentos para formulações pediátricas adequadas.

\section{CONTRIBUIÇÕES}

Todos os autores contribuíram substancialmente na concepção e delineamento do estudo, obtenção, análise e interpretação dos dados, redação e revisão crítica e aprovaram a versão final a ser publicada. 


\section{DECLARAÇÃO DE CONFLITO DE INTERESSES}

Nada a declarar.

\section{FONTES DE FINANCIAMENTO}

Não há.

\section{REFERÊNCIAS}

1. Instituto Nacional de Câncer José Alencar Gomes da Silva [Internet]. Rio de Janeiro: INCA; [data desconhecida]. Câncer infanto-juvenil. [modificado 2019 maio 21; acesso 2019 maio 23]. Disponível em: https://www.inca. gov.br/tipos-de-cancer/cancer-infantojuvenil

2. Instituto Nacional de Câncer José Alencar Gomes da Silva [Internet]. Rio de Janeiro: INCA; [data desconhecida]. Leucemia. [modificado 2018 out. 10; acesso 2019 maio 23]. Disponível em: https://www.inca.gov.br/tipos-decancer/leucemia

3. Schmiegelow K, Nielsen SN, Frandsen TL, et al. Mercaptopurine/Methotrexate maintenance therapy of childhood acute lymphoblastic leukemia: clinical facts and fiction. J Pediatr Hematol Oncol. 2014;36(7):503-17. doi: https://doi.org/10.1097/MPH.0000000000000206

4. Pui $\mathrm{CH}$, Evans WE. A 50-year journey to cure childhood acute lymphoblastic leukemia. Semin Hematol. 2013;50(3):185-96. doi: https://doi.org/10.1053/j. seminhematol.2013.06.007

5. Pui CH, Yang JJ, Bhakta N, et al. Global efforts toward the cure of childhood acute lymphoblastic leukaemia. Lancet Child Adolesc Health. 2018;2(6):440-454. doi: https://doi.org/10.1016/s2352-4642(18)30066-x

6. Bjerknes K, Bøyum S, Kristensen S, et al. Manipulating tablets and capsules given to hospitalised children in Norway is common practice. Acta Paediatr. 2017;106(3):503-508. doi: https://doi.org/10.1111/ apa. 13700

7. Andersson AC, Lindemalm S, Eksborg S. Dividing the tablets for children-good or bad?. Pharm Methods. 2016;7(1):23-7. doi: https://doi.org/10.5530/ phm.2016.7.4

8. Richey RH, Hughes C, Craig JV, et al. A systematic review of the use of dosage form manipulation to obtain required doses to inform use of manipulation in paediatric practice. Int J Pharm. 2017;518(1-2):155-66. doi: https://doi.org/10.1016/j.ijpharm.2016.12.032

9. Conroy S, Newman C, Gudka S. Unlicensed and off label drug use in acute lymphoblastic leukaemia and other malignancies in children. Ann Oncol. 2003;14(1):42-7. doi: https://doi.org/10.1093/annonc/mdg031

10. Mulla H, Buck H, Price L, et al. 'Acceptability' of a new oral suspension formulation of mercaptopurine in children with acute lymphoblastic leukaemia. J Oncol
Pharm Pract. 2016;22(3):387-95. doi: https://doi. org/10.1177/1078155215577808

11. National Institute for Occupational Safety and Health, Centers for Disease Control and Prevention, Department of Health and Human Services. NIOSH Alert: preventing occupational exposure to antineoplastic and other hazardous drugs in health care settings [Internet]. Cincinnati, OH: NIOSH; 2004 [cited 2019 May 23]. Available from: https://www.cdc.gov/niosh/docs/2004165/pdfs/2004-165.pdf

12. National Institute for Occupational Safety and Health. Centers for Disease Control and Prevention, Department of Health and Human Services. NIOSH List of antineoplastic and other hazardous drugs in healthcare settings, 2016 [Internet]. Cincinnati, OH: NIOSH; 2016. [cited 2020 Feb 06]. Available from: https://www.cdc.gov/niosh/docs/2016-161/pdfs/2016161.pdf

13. Crickman R, Finnell D. Systematic Review of control measures to reduce hazardous drug exposure for health care workers. J Nurs Care Qual. 2016;31(2):183-90. doi: https://doi.org/10.1097/NCQ.0000000000000155

14. Böhlandt A, Sverdel Y, Schierl R. Antineoplastic drug residues inside homes of chemotherapy patients. Int J Hyg Environ Health. 2017;220(4):757-765. doi: https:// doi.org/10.1016/j.ijheh.2017.03.005

15. Siden R, Kem R, Ostrenga A, et al. Templates of patient brochures for the preparation, administration and safe-handling of oral chemotherapy. J Oncol Pharm Pract. 2014;20(3):217-24. doi: https://doi. org/10.1177/1078155213484786

16. Laks D, Longhi F, Wagner MB, et al. Avaliação da sobrevida de crianças com leucemia linfocítica aguda tratadas com o protocolo Berlim-Frankfurt-Munique. J Pediatr. (Rio J.). 2003;79(2):149-58. doi: https://doi. org/10.1590/S0021-75572003000200010

17. Bragalone DL. Drug information handbook for oncology. 16th ed. Hudson, Ohio: Wolters Kluwer; 2019-2020.

18. Fotoohi AK, Coulthard SA, Albertioni F. Thiopurines: factors influencing toxicity and response. Biochem Pharmacol. 2010;79(9):1211-20. doi: doi: https://doi. org/10.1016/j.bcp.2010.01.006

19. Ebbesen MS, Nersting J, Jacobsen JH, et al. Incorporation of 6-thioguanine nucleotides into DNA during maintenance therapy of childhood acute lymphoblastic leukemia-the influence of thiopurine methyltransferase genotypes. J Clin Pharmacol. 2013;53(6):670-4. doi: https://doi.org/10.1002/jcph.81

20. Lancaster DL, Patel N, Lennard L, et al. 6-Thioguanine in children with acute lymphoblastic leukaemia: influence of food on parent drug pharmacokinetics and 6-thioguanine nucleotide concentrations. Br J Clin Pharmacol. 2001;51(6):531-9. doi: https://doi.org/10.1046/j.03065251.2001.01391.x 
21. Agência Nacional de Vigilância Sanitária [Internet]. Brasília, DF: ANVISA; [data desconhecida]. Lista de medicamentos de referência; [acesso 2019 maio 23]. Disponível em: http://portal.anvisa.gov.br/registros-eautorizacoes/medicamentos/produtos/medicamentosde-referencia/lista

22. World Health Organization. Model List of Essential Medicines for Children [Internet]. 7th ed. Genève: WHO; 2019 [cited 2020 Feb 06]. Available from: https://www.who.int/medicines/publications/ essentialmedicines/en/

23. European Pharmaceutical Review. [Internet]. United Kingdom: Russell Publishing; 2010-2020. Nova Laboratories secures FDA approval for Xaluprine. [update 2014 May 1; cited 2020 February 06]; [about 1 screens]. Available from: https://www. europeanpharmaceuticalreview.com/news/25344/novalaboratories-secures-fda-approval-xaluprine/

24. Mulla H, Leary A, White P, et al. A step toward more accurate dosing for mercaptopurine in childhood acute lymphoblastic leukemia J Clin Pharmacol. 2012;52(10):1610-3. doi: https://doi. org/10.1177/0091270011423663

25. van Santen E, Barends DM, Frijlink HW. Breaking of scored tablets: a review. Eur J Pharm Biopharm. 2002;53(2):139-45. doi: https://doi.org/10.1016/s09396411(01)00228-4

26. Marriott JL, Nation Roger RL. Splitting tablets. Aust Prescr. 2002;25:133-5. doi: https://doi.org/10.18773/ austprescr.2002.131

27. National Institute for Occupational Safety and Health [Internet]. Cincinnati, OH: NIOSH; [date unknown]. Hazardous drug exposures in health care: antineoplastic agents. [reviewed 2017 Sept 13; cited 2019 May 23]. Available from: https://www.cdc.gov/niosh/topics/ hazdrug/antineoplastic.html

28. Power LA, Coyne JW. ASHP guidelines on handling hazardous drugs. Am J Health Syst Pharm. 2018;75(24):1996-2031. doi: https://doi.org/10.2146/ ajhp 180564

29. Breitkreutz J, Buckham J, Fischer R, et al. Comparative in vitro studies on different 6-mercaptopurine formulations for use in children. Paed Perinat Drug Ther. 2007;8(1):3139. doi: https://doi.org/10.1185/146300907X167817

30. Teixeira MT, Sá-Barreto LCL, Gratieri T, et al. Key technical aspects influencing the accuracy of tablet subdivision. AAPS PharmSciTech. 2017;18(4):13931401. doi: https://doi.org/10.1208/s12249-016-0615-y

31. Habib WA, Alanizi AS, Abdelhamid MM, et al. Accuracy of tablet splitting: Comparison study between hand splitting and tablet cutter. Saudi Pharm J. 2014;22(5):454-9. doi: https://doi.org/10.1016/j. jsps.2013.12.014

32. McDevitt JT, Gurst AH, Chen Y. Accuracy of tablet splitting. Pharmacotherapy. 1998;18(1):193-7. doi: https://doi.org/10.1002/j.1875-9114.1998.tb03838.x

33. Verrue C, Mehuys E, Boussery K, et al. Tablet-splitting: a common yet not so innocent practice. J Adv Nurs. 2011;67(1):26-32. doi: https://doi.org/10.1111/j.13652648.2010.05477.x

34. Teixeira MT, Sá-Barreto LCL, et al. Panorama dos aspectos regulatórios que norteiam a partição de comprimidos. Rev Panam Salud Publica. 2016;39(6):372-77.

35. Borja-Oliveira CR. Pill organizers and pill cutters: risks and limitations. Rev Saude Publica. 2013;47(1):123-7. https://doi.org/10.1590/S0034-89102013000100016

36. Instituto Nacional de Câncer José Alencar Gomes da Silva [Internet]. Rio de Janeiro: INCA; [data desconhecida]. Quimioterapia antineoplásica. [modificado 2018 nov. 14; acesso 2020 mar. 18]. Disponível em: https://www. inca.gov.br/exposicao-no-trabalho-e-no-ambiente/ medicamentos/quimioterapia-antineoplasica

Recebido em 22/11/2019 Aprovado em 24/3/2020 